\title{
THE EFFECTIVE FEATURES FORMATION FOR THE IDENTIFICATION OF REGIONS OF INTEREST IN A FUNDUS IMAGES
}

\author{
N.Yu. Ilyasova ${ }^{1,2}$, R.A. Paringer ${ }^{1,2}$, A.V. Kupriyanov ${ }^{1,2}$, N.S. Ushakova ${ }^{2}$ \\ ${ }^{1}$ Image Processing Systems Institute - Branch of the Federal Scientific Research Centre "Crys- \\ tallography and Photonics" of Russian Academy of Sciences, Samara, Russia \\ ${ }^{2}$ Samara National Research University, Samara, Russia
}

\begin{abstract}
The effective features formation for the identification of regions of interest (ROIs) in a fundus images is proposed. Source texture features are derived from 6 different statistical image descriptors computed by library MaZda. The features are derived respectively from image histogram, image gradient, run-length matrix, co-occurrence matrix, autoregressive model, wavelet transform, each calculated for up to 16 predefined ROIs. The first step of the research is a selecting from this enormous amount of features a subset of the most informative ones for our class of the biomedical images, which provide a maximum value of group separability criterion of discriminative analysis. New effective features set is formed from selected features with using discriminative analysis technique. In order to evaluate the separability quality, we calculated the clusterization error for each features set and various fragmentation window sizes. We use a K-means clusterization method and the Euclidean and Mahalanobis distance as a similarity measure. The required minimum size of a fragmentation window and the similarity measure were selected according to the criterion of the minimum clusterization error.
\end{abstract}

Keywords: fundus images, laser coagulation, texture analysis, discriminative analysis.

Citation: Ilyasova NYu, Paringer RA, Kupriyanov AV, Ushakova NS. The effective features formation for the identification of regions of interest in a fundus images. CEUR Workshop Proceedings, 2016; 1638: 788-795. DOI: 10.18287/1613-0073-2016-1638-788-795

\section{Introduction}

Computer analysis of images became the basic tool of medical systems allowing one to increase quality of treatment significantly. Information technologies are most widely introduced in ophthalmology $[1,2]$. The main reason for the irreversible blindness among employable population in developed countries is diabetic retinopathy (DR). At diabetic retinopathy all parts of the eye's retina are damaged, however, in particular, 
changes in central parts in the form of the diabetic macula edema may cause the quickest and the most irreversible visual decrement [3] (Fig.1). Treatment of the diabetic macula edema is rather a complicated process including both conservative and surgical laser methods. Laser coagulation of the eye's retina is "the golden standard" for medical treatment, the effectiveness of which has been proved during a large-scale study (ETDRS, 1987) [4]. During laser treatment a series of metered microscopic thermal wounds - laser coagulates - are applied in the edema zone on the eye's retina. Coagulates are overlayed either one by one or in series located in the form of a specified regular-shaped figure - a pattern, or with a preliminarily planned location of coagulates followed by the obtained plan to be overlayed onto a retina image in online mode [5]. The optimum location of coagulates is most preferable, that means that they are to be located in the edema zone at maximum equal distances between each other, and their intrusion onto vessels are avoided. Preliminarily planned coagulates shall be overlayed by an automatic beam positioning control system that allows to give medical treatment with high accuracy (Fig.2) [6, 7].
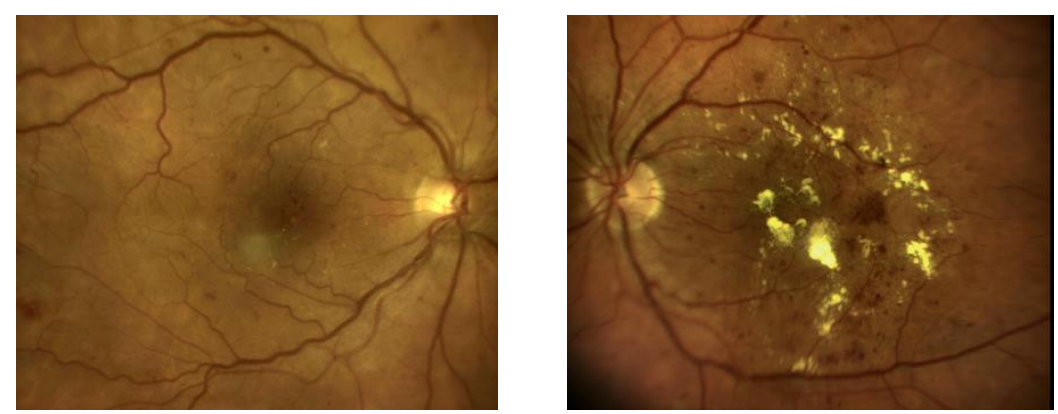

Fig. 1. The example of a fundus diagnostic image without pathology (left) and with pathology detected (right)

The main disadvantage of this approach is that there is no optimum location of coagulates in conditions of high diversity of edema forms and retinal vascular patterns therein. First, this is due to a limited choice of pattern's forms which often correspond neither to the edema form nor to a status of vessels. If the arrangement is conducted manually only by coagulate, their optimal position will be experience based and more time will be required for planning [8].

Thus, the development of the information technology, including methods and algorithms for optimal automatic coagulate filling in the defined edema zone with different arrangements of blood vessels therein, is currently a critical task.

To computerize a laser coagulation procedure it is necessary to make the image segmentation for particular ranges of interest which are characterized by the presence of four classes of objects, i.e. exudates, blood vessels, intact sectors and the macula. 


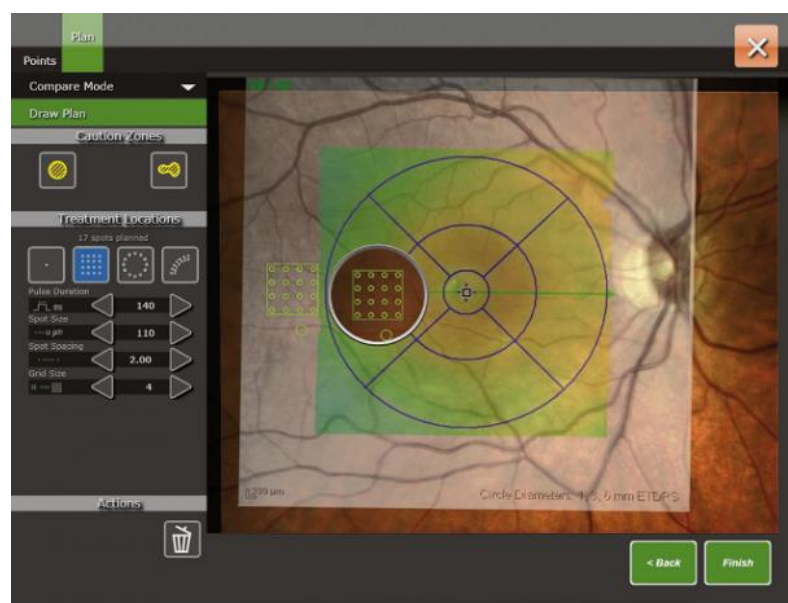

Fig. 2. Examples of patterns NAVILAS [7]

The macular edema region is to be defined by aggregated exudation zones. During laser therapy it is recommended by doctors to avoid the zone of blood vessels, and it is strongly forbidden to overlay coagulates on the macula zone [8].

\section{The technique of defining ranges of interest based on the texture analysis of biomedical images}

The segmentation is to be performed by making a decision on the membership of fragmented zones to one of the four given classes of object images. The fragmentation was carried out by dividing the image into several square-shaped blocks, which were classified using the technique of selecting for the effective recognition features (Fig. 3).

At the initial stage, the technique is used to select proper-sized fragments and to preliminarily classify them, thus involving medical practitioners to provide the recognition system training. The analysis of the fragments has shown that they may differ well enough by their textural characteristics. Textural features have shown good results in recognizing biomedical images and their further diagnosis [9 - 11]. We used the known MaZda library which allows us to calculate up to 300 different texture features $[12,13]$. Different texture features such as correlation, homogeneity, short run emphasis, long run emphasis, run percentage and many more were extracted from the digital fundus images. Texture features derived from 6 different statistical image descriptors can be computed by MaZda. The features are derived respectively from image histogram, image gradient, run-length matrix, co-occurrence matrix, autoregressive model, wavelet transform, each calculated for up to 16 predefined ROIs.

The effectiveness of the obtained feature set was evaluated based on the discriminative analysis [14-18]. The purpose of the research is to select, from this enormous amount of features, a subset of the most informative ones for our class of biomedical images, which provide a minimum clusterization error. It is necessary herewith to 
define the best dimension of a mask, by means of which the system will process the image and make the automatic selection of ranges of interest during the laser coagulation procedure.

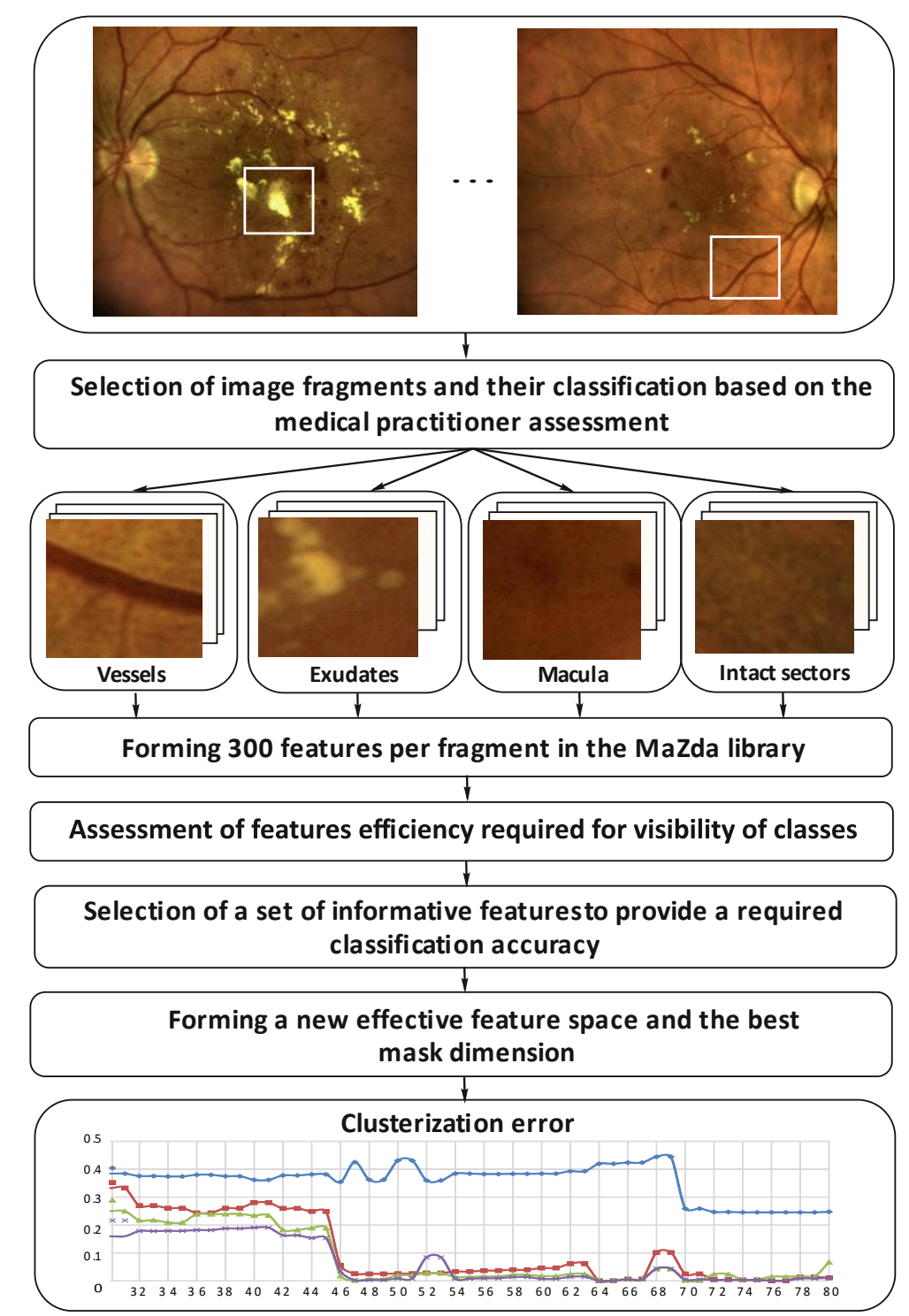

Fig. 3. The technique of forming the effective features for the identification of ranges of interest in fundus images

As follows from the discriminative analysis, the best features have been identified for each sample of objects based on a separability criterion. In order to evaluate the separability quality, we calculated the clusterization error for each feature set and various 
fragmentation window sizes. Feature sets were formed through selecting the best ones, based on values of individual separability criteria. We used a K-means clusterization method in the discriminative analysis, and the Euclidean and Mahalanobis distance was used as a similarity measure [14]. The required minimum size of a fragmentation window and the similarity measure were selected according to the criterion of the minimum clusterization error.

\section{Experimental research findings}

Experimental researches were carried out on samples formed while analyzing 70500 fragments, which contained different image classes. We used 70 retinal fundus images in our analysis. The researches were aimed at selecting the best feature set and the fragmentation window to identify ranges of interest with a prescribed accuracy. Specific characteristics of the analyzed diagnostic image thereby impose restrictions on the size of the fragmentation window. The smaller the window's size, the highest segmentation quality obtained during laser coagulation. Therefore, while analyzing relationships obtained during the researches, we have selected the smallest value of the fragmentation window, at which there is a quantum leap in values of the clusterization error and the separability criterion. Fig. 4 shows the interrelationship of values of the separability group criterion and the fragmentation size at various amounts of selected features characterized by the maximum individual separability criterion.

The experiments have shown that the largest group separability criterion is possessed by sets of 16-20 features when the minimum fragmentation window size is 46 pixels (Fig.4).

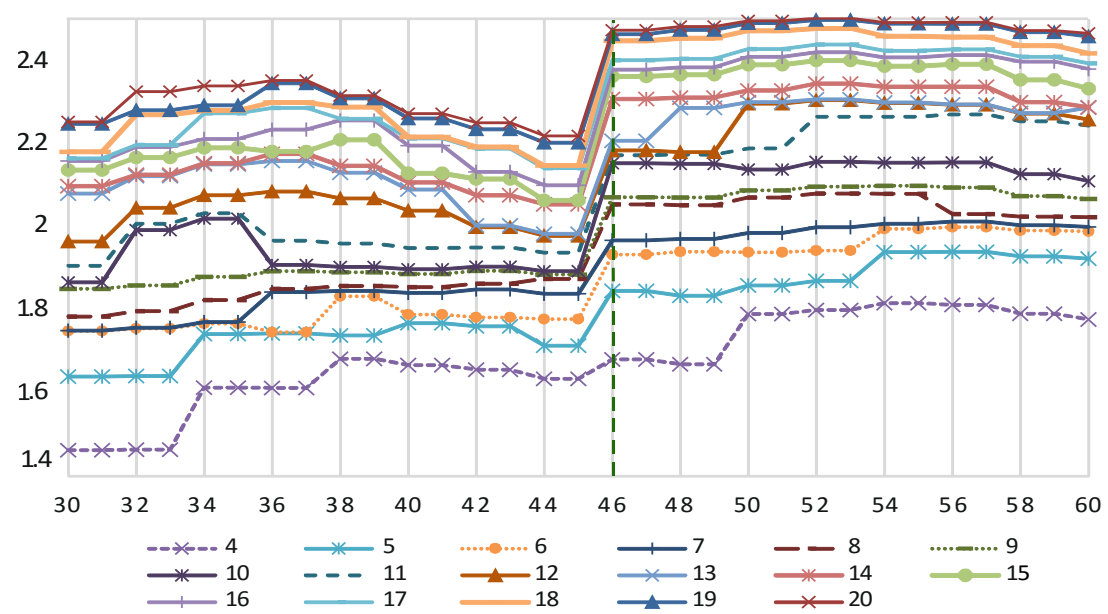

Fig. 4. The interrelationship of values of the group separability criterion and the fragmentation size at various amounts of selected features with the maximum individual separability criterion

The specified sets are thereby characterized with their close interrelationships. If we consider the interrelationship of the clusterization error and fragmentation sizes for 
five lowest-dimensional feature sets, i.e. 16-20 (Fig.5), it can be observed that the least error with the fragmentation window 46 pixels in size is possessed by the set of 18 features.

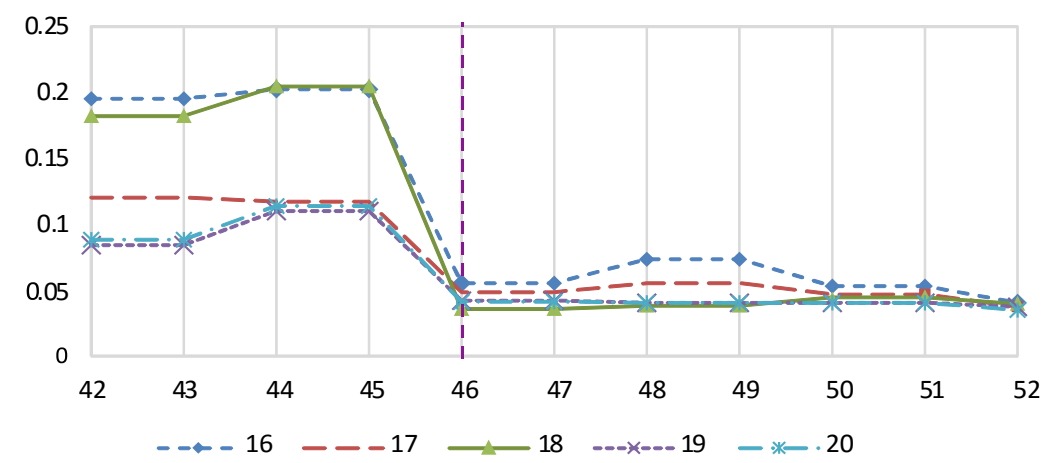

Fig. 5. The interrelationship of the clusterization error and the fragmentation window size when using the Mahalanobis similarity measure and the sets of 16, 17, 18, 19 and 20 features with the maximum separability criterion

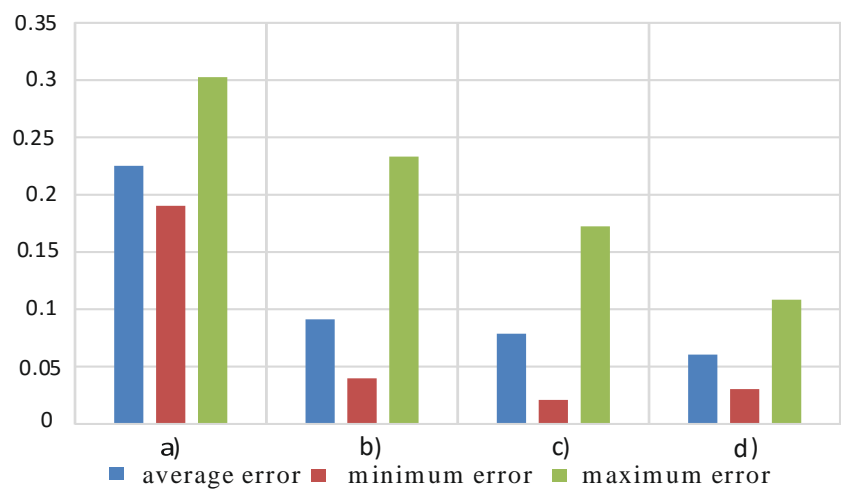

Fig. 6. Illustration of various types of the clusterization error depending on the types of features and various similarity measures: a) Euclidean distance, original features; b) Mahalanobis distance, original features c) Euclidean distance and set of newly formed features; d) Mahalanobis distance and set of newly formed

From Fig. 6, which illustrates the interrelationship of maximum, average and minimum clusterization errors depending on the type of features (the subset of original features MaZda with the maximum separability criterion and newly formed features based on the discriminative analysis) and the type of similarity measures, and from Fig. 7 it may be concluded that the best clusterization result may be provided by the Mahalanobis distance and the set of newly formed features obtained from 18 original features. It is thereby recommended to use the fragmentation window 46 pixels in size. This shall provide at least $95 \%$ of identification certainty of ranges of interest. 




Fig. 7. The interrelationship of the clusterization error and the fragmentation window size at various similarity measures when using 18 features with the maximum separability criterion: a) Euclidean distance, original features; b) Mahalanobis distance, original features c) Euclidean distance, set of newly formed features; d) Mahalanobis distance, set of newly formed features

\section{Conclusion}

The effective features formation for the identification of regions of interest (ROIs) in a fundus images is proposed during laser coagulation is proposed. The method is based on the texture analysis of selected image patterns. The analysis of informative value of obtained feature space and the selection of the most effective features is performed using the data discriminative analysis. The best values of image fragmentation dimensions for the image segmentation and the feature sets providing the precise identification required for regions of interest are determined herein. Further researches shall be aimed at the improvement of individual stages of the technology presented herein, particularly, shape modifications of the fragmentation window, at the use of the image preprocessing procedure, which enables to focus on fundus image elements required for the analysis, and the development of an alternative feature selection method and the use of a more sophisticated clusterization algorithm.

\section{Acknowledgements}

The work has been performed with partial financial support from the Ministry of Education and Sciences of the Russian Federation within the framework of implementation of the Program for Improving the SSAU Competitiveness among the World's Leading Research and Educational Centers for the Period of 2013-2020s; under the RFBR grants (the Russian Foundation for Basic Researches) 14-07- 97040, 15-2903823, 15-29- 07077, 16-57-48006; within the Basic Research Program No. 6 ONIT RAN of the Russian Academy of Sciences "Mathematical Methods and Information Technologies for the Analysis of Biomedical Images in Medical Diagnostics Applications" 2016 


\section{References}

1. Ilyasova NYu. Computer Systems for Geometrical Analysis of Blood Vessels Diagnostic Images. Optical Memory and Neural Networks (Information Optics), 2014; 23(4): 278-286.

2. Ilyasova $\mathrm{NYu}$. Methods for digital analysis of human vascular system. Literature review. Computer Optics, 2013; 37(4): 517-541. [In Russian]

3. Shadrichev FE. Diabetic retinopathy. Modern optometry, 2008; 4: 36. [In Russian]

4. Astakhov YS, Shadrichev FE, Krasavina MI, Grigoryeva NN. Modern approaches to the treatment of a diabetic macular edema. Ophthalmologic sheets, 2009; 4: 59-69. [In Russian]

5. Kernt M, Cheuteu R, Liegl RG, et al. Navigated focal retinal laser therapy using the NAVILAS system for diabetic macula edema. Ophthalmology, 2012; 109: 692-700.

6. Chhablani J, Kozak I, Barteselli G, El-Emam S. A novel navigated laser system brings new efficacy to the treatment of retinovascular disorders. Oman Journal of Ophthalmology, 2013; 6 (1): 18-22.

7. Navilas ${ }^{\circledR}$ Navigated PRP 4: https://www.youtube.com/watch?v=mtMOYdIuyvI

8. Zamytsky EA. Laser treatment of a diabetic macular edema. Postgraduate bulletin of the Volga region, 2015; 1-2: 79. [In Russian]

9. HeiShun Yu, Tischler B, Qureshi MM, Soto JA, Anderson S, Daginawala N, Li B, Buch K. Using texture analyses of contrast enhanced CT to assess hepatic fibrosis. European Journal of Radiology, 2016; 85(3): 511-517.

10. Acharya UR, Ng EY, Tan JH, Sree SV, Ng KH. An integrated index for the identification of diabetic retinopathy stages using texture parameters. Journal of Medical Systems, 2012; 36(3): 2011-2020.

11. Kutikova VV, Gaidel AV. Study of informative feature selection approaches for the texture image recognition problem using Laws' masks. Computer Optics, 2015; 39(5): 744-50 [In Russian]. DOI: 10.18287/0134-2452-2015-39-5-744-750.

12. Strzelecki M, Strzelecki M, Szczypinski P, Materka A, Klepaczko A. A software tool for automatic classification and segmentation of 2D/3D medical images. Nuclear Instruments \& Methods In Physics Research Section A: Accelerators, Spectrometers, Detectors and Associated Equipment, 2013; 702: 137-140.

13. Szczypiński PM, Strzelecki M, Materka A, Klepaczko A. MaZda-A software package for image texture analysis, Computer Methods and Programs in Biomedicine, 2009; 94(1): 6676.

14. Fukunaga K. Introduction to statistical pattern recognition. New York and London: Academic Press, 1972. 369 p.

15. Ilyasova NYu, Kupriyanov AV, Paringer RA. Formation of features for improving the quality of medical diagnosis based on discriminant analysis method. Computer Optics, 2014; 38(4): 751-756. [In Russian]

16. Kim JA, Myuller ChU, Klekka WR. Factor, discriminant and cluster analysis. Moscow, "Financy I Statistica” Publisher, 1989. 215 p.

17. Ilyasova NYu, Kupriyanov AV, Paringer RA. The Discriminant Analysis Application to Refine the Diagnostic Features of Blood Vessels Images. Optical Memory \& Neural Networks (Information Optics), 2015; 24(4): 309-313.

18. Ilyasova NYu, Paringer RA. Research effectiveness of features for the vascular pathologies diagnosis. Scientific Journal of "Proceedings of the Samara Scientific Center of the Russian Academy of Sciences", Samara Scientific Center of the Russian Academy of Sciences, 2015; vol.17, N2(5): 1015-1020. [In Russian] 